\title{
Die schwedische Integrationspolitik
}

Schweden wird in internationalen Vergleichsstudien immer wieder für seine Integrationspolitik gepriesen. Dieses positive Urteil spiegelt sich allerdings nicht bei der Integration von Migranten auf dem Arbeitsmarkt in Bezug auf Beschäftigungsrate und Lohnniveau wider. Tatsächlich bleibt Schweden hier hinter den Ansprüchen eines universalistischen Modells zurück. Ursachen hierfür sind neben Diskriminierungen seitens der Arbeitgeber auch verfehlte Maßnahmen in der Integrationspolitik.

\section{Einleitung}

Die international so viel gepriesene schwedische Integrationspolitik weist bei genauerer Betrachtung der Praxis zahlreiche Defizite auf. Im Ausland Geborene haben einen geringeren Beschäftigungsgrad, eine höhere Arbeitslosigkeit und niedrigere Löhne als gebürtige Schweden. Die universalistische Zielvorstellung der Integrationspolitik - gleiche Rechte, Möglichkeiten und Pflichten - wird bei Weitem verfehlt. Die Integrationspolitik ist jedoch seit jeher ein blinder Fleck im schwedischen Modell. Das Vollbeschäftigungsziel war zwar zunächst nur durch einen Zuzug von Arbeitskräften zu realisieren. Doch fehlte es an politischen

\section{Abb. 1: Bevölkerungsanteil mit Hochschulausbildung unter Immi- granten und im Inland Geborenen (Basisjahr 2000) - in \% -}

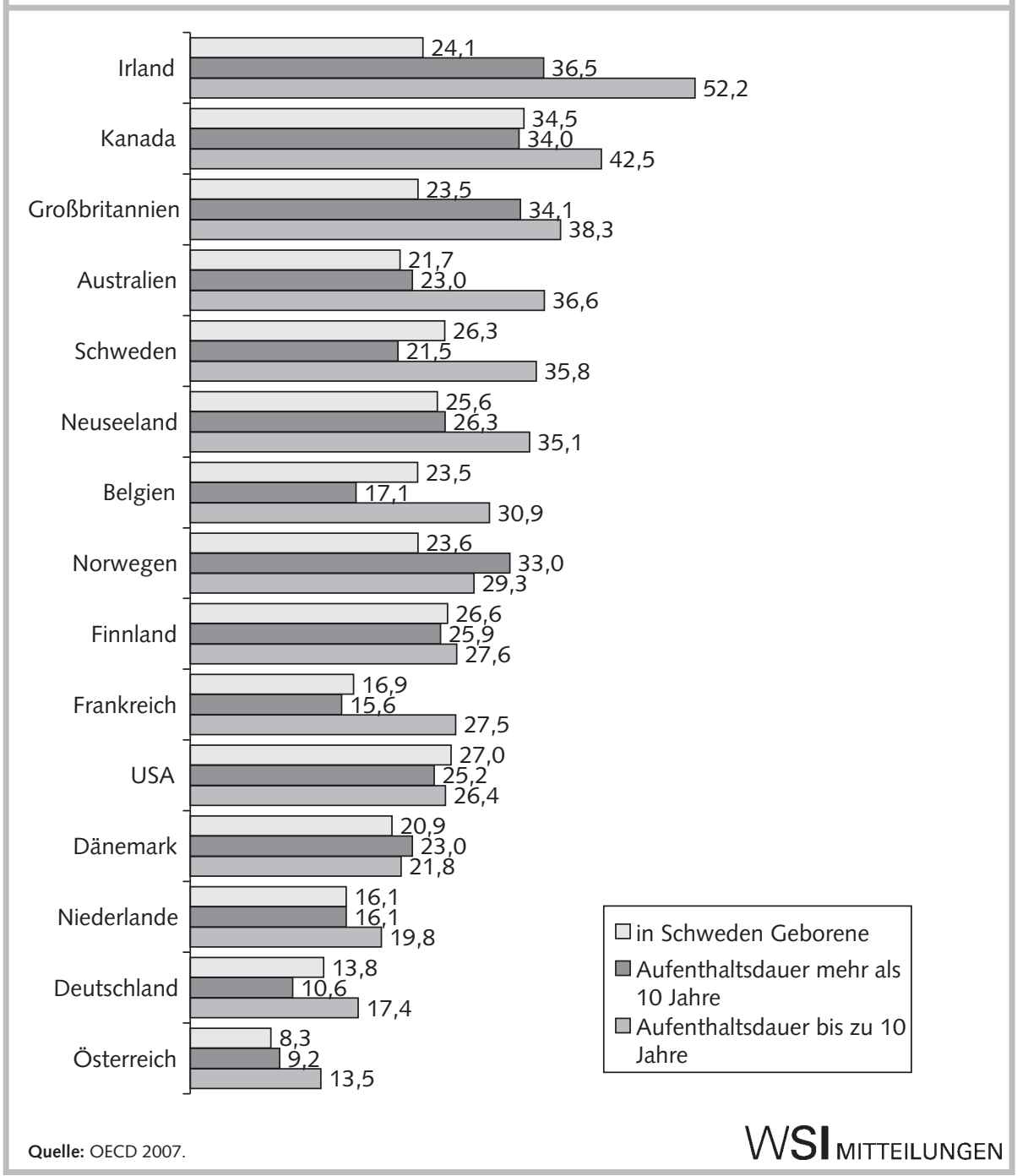

Maßnahmen, um ihre Integration zu fördern.

Der vorliegende Artikel gibt einen kritischen Überblick über die möglichen Gründe für die bestehenden Unterschiede im Lohnniveau und bei den Beschäftigungsmöglichkeiten zwischen einheimischen Schweden und Immigranten. Darüber hinaus werden Überlegungen angestellt, wie die Integration in den Arbeitsmarkt verbessert werden kann.

\section{Zuwanderung in Schweden}

Rund $13 \%$ der Schweden im arbeitsfähigen Alter sind im Ausland geboren, das entspricht in etwa dem Migrantenanteil in Deutschland. Eine weitere Ähnlichkeit zwischen Deutschland und Schweden besteht darin, dass der im Ausland geborene Teil der Bevölkerung äußerst heterogen zusammengesetzt ist: Er besteht aus eingewanderten Arbeitskräften und Flüchtlingen sowie deren Angehörigen. Nach dem Zweiten Weltkrieg kam es zu einer relativ breiten Einwanderung von Arbeitskräften nach Schweden, die bis in die 1970er Jahre fortdauerte. Es gab aber zu keinem Zeitpunkt

Lena Schröder, Associate Professor für Wirtschaftsgeschichte, war Forschungsdirektorin bei der Schwedischen Integrationsbehörde. Gegenwärtig ist sie Dozentin am Linnaaeus Center for Integration Studies (SULCIS) der Universität Stockholm und am Swedish Institut for Social Research (SOFI). Arbeitsschwerpunkte: Integration und Immigrationsforschung, Ökonomie des Arbeitsmarktes. e-mail: lena.schroder@sofi.su.se)

Übersetzung aus dem Schwedischen von Adolf Krämer 
eine aktive Anwerbepolitik von „Gastarbeitern". Die eingewanderten Arbeitskräfte bekamen unbefristete Aufenthaltsgenehmigungen und es gab keinerlei Restriktionen hinsichtlich der Berufe, Branchen oder Regionen, in denen sie und ihre Angehörigen arbeiten durften (Widgren 1987).

Nach den Ölkrisen der 1970er Jahre bestand die nichtnordische Einwanderung nach Schweden hauptsächlich aus Flüchtlingen und deren Angehörigen. Von der heutigen Bevölkerung mit Migrationshintergrund sind die meisten in Finnland (fast 180.000) oder im früheren Jugoslawien (mehr als 140.000) geboren. Weitere wichtige Gruppen sind im Irak (fast 100.000), in Polen (mehr als 50.000) und im Iran (fast 60.000) geborene Menschen (Statistiska centralbyrån 2008, S. 119).

Besonders hervorzuheben ist der hohe Anteil an Hochschulabsolventen unter Flüchtlingen und ihren Angehörigen, besonders bei denen, die in den letzten Jahren gekommen sind. Der Anteil an Akademikern entspricht ungefähr dem in Australien und Neuseeland, also Ländern, die aktiv hochqualifizierte Arbeitskräfte aus aller Welt anwerben.

\section{Integrationspolitik in Schweden}

\subsection{INSTITUTIONELLE STRUKTUREN}

Die institutionelle Zuständigkeit für Einwanderungs- und Integrationsfragen hat in Schweden häufig gewechselt. Eine Veränderung, die bis heute fortwirkt, gab es Mitte der 1980er Jahre, als die Zuständigkeit für neu angekommene Flüchtlinge von der Arbeitsmarktbehörde zur Einwanderungsbehörde und in die Kommunen verlegt wurde. Mit dieser Reorganisation änderte sich die Perspektive von einer Arbeitsmarktorientierung mit enger Anbindung an die Arbeitsmarktpolitik zu einer Perspektive der Fürsorge als Teil der Sozialpolitik (Edin et al. 2004).

Eine weitere Veränderung erfolgte 1997, als die Einwanderungsbehörde in zwei Teile aufgeteilt und eine neu gebildete Integrationsbehörde mit Fragen der Integration betraut wurde. Die generelle Zielsetzung für die schwedische Integrationspolitik blieb dabei unverändert: gleiche Rechte, Möglichkeiten und Pflichten für alle, un- abhängig von ihrem nationalen und ethnischen Hintergrund. Dieses universalistische Prinzip soll deutlich machen, dass Integration keine Einbahnstraße ist. Nicht nur die, die nach Schweden einwandern, sollen sich an die schwedische Gesellschaft anpassen, sondern auch die schwedische Gesellschaft muss sich darauf einstellen, dass ein wachsender Anteil ihrer Mitbürger seine Wurzeln in anderen Ländern hat. Die Integrationsbehörde wurde schließlich 2007 geschlossen und die Zuständigkeiten wurden auf mehrere andere staatliche Institutionen verteilt.

Ein Standardelement der Integrationspolitik seit Mitte der 1960er Jahre ist Sprachunterricht. Heutzutage haben alle Personen, die keine grundlegenden Kenntnisse der schwedischen Sprache haben, das Recht auf kostenlosen Schwedischunterricht. Es gibt drei verschiedene Studienwege, deren Dauer und Inhalt abhängig sind von den individuellen Voraussetzungen der Schüler. Neu angekommene Flüchtlinge und deren Angehörige haben das Recht, an einem ca. zweijährigen Einführungsprogramm teilzunehmen, das die Kommunen von der Arbeitsmarktverwaltung übernommen haben und seit Anfang der 1990er Jahre mit staatlicher Unterstützung durchführen. Über den Unterricht in der schwedischen Sprache hinaus soll das Programm eine Orientierung in der schwedischen Gesellschaft und Arbeitswelt bieten. Diese Einführung folgt einem individuellen Lehrund Lernplan, der gemeinsam durch den Flüchtling und den Sachbearbeiter der Kommune erstellt wird.

Ein prominentes Merkmal der schwedischen Integrationspolitik besteht darin, dass keine besonderen Maßnahmen für Einwanderer angeboten werden. Im Grundsatz sind sämtliche Politikprogramme sowohl für Einwanderer als auch für in Schweden geborene Personen zugänglich. Mit Ausnahme gewisser Programme für Neuankömmlinge gibt es beispielsweise keine besonderen Arbeitsmarktprogramme für Einwanderer. Die meisten, denen Asyl gewährt wird, besitzen von Anfang an dauerhafte Aufenthaltsgenehmigungen. Und es gibt keinerlei Beschränkungen für sie und ihre Angehörigen hinsichtlich des Wohnortes oder der Arbeitsaufnahme.

Zur Integrationspolitik gehört auch die Antidiskriminierungspolitik. Sie fällt in die Zuständigkeit eines Ombudsmannes für Diskriminierung. Diese Einrichtung datiert aus dem Jahre 1986 und hat den Auf- trag, Diskriminierung aufgrund ethnischer Zugehörigkeit, Religion oder anderer Glaubensauffassungen entgegenzuwirken. Ein spezielles Gesetz gegen ethnische Diskriminierung im Arbeitsleben trat 1999 in Kraft. Es ist seitdem in mehreren Schritten ergänzt und verstärkt worden.

Laut einem Index, der mit Teilfinanzierung der Europäischen Union (EU) vom British Council und der Migration Policy Group (2007) erarbeitet wird, erfüllt die schwedische Integrationspolitik die Konventionen des Europarates und der verschiedenen Erklärungen der EU. In dieser Beurteilung wird der schwedischen Integrationspolitik der erste Platz unter den 28 verglichenen Ländern zugewiesen.

\subsection{DIE POLITISCHE PRAXIS}

Die Tatsache, dass die institutionellen Voraussetzungen existieren, bedeutet noch lange nicht, dass die Integrationspolitik auch in der Praxis erfolgreich ist. Es gibt zahlreiche Forschungsergebnisse, die belegen, dass im Ausland geborene Menschen eine geringere Beschäftigungsquote und eine höhere Arbeitslosigkeit aufweisen als im Inland geborene. Der Unterschied ist am größten bei denen, die gerade erst nach Schweden gekommen sind. Er bleibt aber auch bei denjenigen, die schon viele Jahre im Land wohnen. Unabhängig von der Aufenthaltsdauer in Schweden, dem Alter und dem Niveau der Ausbildung weisen Personen aus außereuropäischen Ländern einen geringeren Beschäftigungsgrad und höhere Arbeitslosigkeit auf als Personen, die in anderen Teilen der Welt geboren sind (Schröder 2007).

Inwieweit die Integration auf dem Arbeitsmarkt in Schweden schlechter ist als in anderen Ländern, ist schwer zu beurteilen. Internationale Statistiken weisen nur die heterogenen Kategorien „im Ausland Geborene" oder „ausländische Bürger" aus, was je nach Land Unterschiedliches bedeuten kann. Hier werden Äpfel mit Birnen verglichen. In einem Teil der Länder sind die im Ausland geborenen Personen in der Hauptsache Neuankömmlinge. In anderen Ländern haben sie schon eine lange Zeit im Einwanderungsland gewohnt. In einem Teil der Länder sind die Einwandernden in der Hauptsache Arbeitsmigranten, wobei die Bedingung für die Aufenthaltsgenehmigung ein Arbeitsverhältnis ist. Oder es handelt sich um Personen, die für den Arbeitsmarkt besonders attraktiv sind. Eine 
andere Gruppe von Ländern wiederum nimmt hauptsächlich Flüchtlinge und deren Angehörige auf. In den englischsprachigen Ländern sprechen viele Einwanderer die Sprache des neuen Landes als Muttersprache oder als Zweitsprache.

In einer besonderen Studie zur Integration auf dem Arbeitsmarkt in Deutschland hat die OECD kürzlich eine differenziertere Statistik erarbeitet, die u.a. auch Schweden umfasst. Daraus geht hervor, dass die Unterschiede auf dem Arbeitsmarkt zwischen im Ausland geborenen Personen und solchen, die im Inland geboren sind, in Schweden größer sind als in Deutschland, zumindest was die männlichen Personen angeht (OECD 2005).

\section{Ursachen für Unterschiede auf dem Arbeitsmarkt}

Die großen Unterschiede zwischen Einheimischen und im Ausland geborenen Personen auf dem Arbeitsmarkt deuten darauf hin, dass es der schwedischen Integrationspolitik nicht gelungen ist, ihre oben skizzierten Gleichheitsziele zu erreichen. Hier stellt sich die Frage nach den Ursachen dafür, dass Migranten auf dem Arbeitsmarkt Schwierigkeiten haben.

\subsection{DIE ANGEBOTSSEITE}

Die Forschung war früher in der Hauptsache darauf ausgerichtet, die Erklärungen auf der Angebotsseite des Arbeitsmarktes zu suchen. Den Einwanderern wurden verschiedene Defizite zugeordnet, beispielsweise schlechte Ausbildung, schlechte Kenntnisse der schwedischen Sprache, fremde Kulturen und Sitten. Seit Informationen über die Ausbildung im Ausland geborener Personen in schwedischen Datenbanken zugänglich sind, haben ökonometrische Studien gezeigt, dass das Ausbildungsniveau die Unterschiede auf dem Arbeitsmarkt zwischen Einheimischen und Migranten nicht erklären kann (Schröder 2007).

Kenntnisse der schwedischen Sprache sind allerdings wichtig für den Erfolg auf dem Arbeitsmarkt. Es gibt jedoch keine detaillierten Informationen darüber, wie gut die eingewanderte Bevölkerung schwedisch spricht. Die wenigen vorliegenden Untersuchungen zeigen höchst unterschied- liche Resultate. ${ }^{1}$ Darüber hinaus existiert eine stark positive Korrelation zwischen guten Kenntnissen der schwedischen Sprache und Chancen auf dem Arbeitsmarkt. Für die Forscher ist die wahrscheinlichste Erklärung, dass gute Kenntnisse im Schwedischen zu besseren Beschäftigungschancen führen. Die Ergebnisse lassen jedoch auch die Möglichkeit des Umkehrschlusses $\mathrm{zu}$, nämlich, dass diejenigen, die Arbeit haben, über bessere Möglichkeiten verfügen, schwedisch zu lernen.

Eine andere wichtige Frage in diesem Kontext gilt dem Suchmuster arbeitsloser Individuen nach einer Beschäftigung. Hier liegt nur eine empirische Studie vor, die die Intensität der Arbeitssuche bei Personen aus Ländern außerhalb von Europa vergleicht mit der von einheimischen Schweden. Das Ergebnis ist: Die außerhalb von Europa geborenen Personen suchen Arbeit mit mindestens der gleichen Intensität wie in Schweden geborene (Arai et al. 1999). Der Zugang zu persönlichen Netzwerken ist für die Personen bei der Suche jedoch von großer Bedeutung. Trotz der Vorschrift, sämtliche freien Stellen bei der Arbeitsvermittlung zu melden, wird ein großer Teil der freien Arbeitsplätze durch informelle Rekrutierungskanäle besetzt. Der Zugang eines Individuums zu potenten Netzwerken hat demnach eine große Bedeutung für die Chance, einen Arbeitsplatz zu finden. Arbeitsuchende mit ausländischer Herkunft haben kleinere und schlechtere Netzwerke und können so seltener Arbeit durch persönliche Kontakte bekommen als in Schweden geborene (Olli-Seggendorf 2005; Behtoui 2006a).

\subsection{KULTUR UND RELIGION}

Die Unterschiede auf dem Arbeitsmarkt, die nicht mit den üblichen Variablen des Humankapitals erklärt werden können, sind auch als Resultat kultureller Unterschiede gedeutet worden. Diese Unterschiede, so glaubt man, bestehen darin, dass Personen mit außereuropäischer Herkunft auf modernen westlichen Arbeitsmärkten eine vergleichsweise geringere Produktivität aufweisen. Nur wenige Forschungsansätze haben versucht, den Zusammenhang zwischen Kultur und Produktivität am Arbeitsmarkt zu untersuchen. Die Resultate sind alles andere als überzeugend (Broomé et al. 1996; Scott 1999; Rosholm et al. 2001).

In der internationalen Forschungslandschaft gibt es seit Samuel Huntingtons
(2002) These vom Kampf der Kulturen verstärkt Ansätze, die von einer Unverträglichkeit des Islam mit den westlichen Gesellschaften ausgehen. Auf Schweden bezogen fehlen vergleichbare Untersuchungen. Die Ausgangshypothese dieser Forschungsrichtung ist die Annahme, dass Muslime generell andere Werte haben als die Bevölkerung in Ländern mit christlicher Tradition.

Da vermutlich islamfeindliche Tendenzen in Schweden ebenso verbreitet sind wie in anderen Ländern, scheint es wichtig, diese Forschungsperspektive kritisch zu hinterfragen. Einige dieser Studien nehmen den internationalen Terrorismus als Ausgangspunkt für ihre Fragestellung und verwenden Daten darüber, wie die Einstellung zu Religion und die Identifikation mit dem Heimatland respektive dem Einwanderungsland sich unterscheiden bei Einwanderern mit verschiedenen Religionen und verschiedenen Zeitpunkten der Einwanderung. Ihre Resultate zeigen, dass Muslime länger an ihrer Religion und ihren Traditionen festhalten als Einwanderer anderer Glaubensrichtungen. Obwohl sich in ihren Daten nichts findet, das den Zusammenhang zwischen Religion und Terrorismus aufzeigen könnte, wird dennoch zumindest in einer Studie eine diesbezügliche Schlussfolgerung gezogen (Bisin et al. 2007). In einer anderen wird der Zusammenhang zumindest suggeriert, die Schlussfolgerung aber dem Leser überlassen (Zimmermann et al. 2006).

Darüber hinaus sind die Studien mit ernsten methodologischen Problemen behaftet. An sich besteht unter Wissenschaftlern Konsens darüber, dass man Panelstudien braucht, in denen man die Individuen über eine Anzahl von Jahren beobachtet, um die Prozesse im Zeitablauf studieren zu können. Die Daten, die man in oben genannten Untersuchungen zugrunde gelegt hat, sind jedoch Querschnittsdaten, die sich lediglich auf Migranten mit einjähriger Aufenthaltsdauer beziehen.

Eine andere Studie (Constant et al. 2006a) geht davon aus, dass es Unterschiede in der Arbeitsmoral zwischen Muslimen und Menschen anderer Glaubensrichtungen gibt. Auch hierbei wurden Quer-

\footnotetext{
Die Spannbreite der vorliegenden Untersuchun gen reicht von einem Anteil mit guter Kenntnis der schwedischen Sprache von $34 \%$ bis zu ganzen 88 \% (Rooth/Aslund 2006).
} 
schnittsdaten verwendet. Es wird kein direkter Vergleich zwischen Muslimen und Christen vorgenommen. Ebenso wenig werden Angaben über die individuelle Anbindung an den Arbeitsmarkt gemacht. Stattdessen wird gefragt, wie verschiedene Hintergrundfaktoren (Geschlecht, Ausbildung, Alter bei der Einwanderung) das ethnische Selbstbewusstsein beeinflussen. Eine sehr weitgehende Auslegung dieser Unterschiede wird dann für die Behauptung herangezogen, die Integration von Muslimen sei per se ein Problem. Es gelte, ihre Anpassung an das Einwanderungsland $\mathrm{zu}$ verbessern. Ein empirischer Zusammenhang zwischen Religion und Arbeitsmoral - der Ausgangspunkt des Arguments - wird nicht hergestellt.

In einer späteren Studie desselben Autors (Constant et al. 2006b) wird die Bedeutung, die die ethnische Identifikation für die Anbindung des Individuums an den Arbeitsmarkt hat, untersucht. Die Ergebnisse zeigen, dass muslimische Männer, ebenso wie Männer aller anderen Glaubensrichtungen, mit höherer Wahrscheinlichkeit eine Arbeit finden als Männer ohne religiöse Glaubensüberzeugung. Und zum Erstaunen der Verfasser hat die muslimische Glaubensrichtung (oder irgendeine andere Religion) keinerlei Bedeutung für die Wahrscheinlichkeit, dass Frauen arbeiten.

Letztendlich wird bei dieser Art Studien das Problem immer auf der Angebotsseite, bei den Einwanderern, gesehen. Diejenigen, die nicht in Kultur und Sprache des Einwanderungslandes investieren wollen, haben eine schlechtere Position auf dem Arbeitsmarkt als die, die sich an die neue Gesellschaft anpassen wollen. Dabei rückt die Art und Weise, wie das Einwanderungsland die Einwanderer empfängt und behandelt aus dem Blickfeld. Dies scheint keine Bedeutung für die Identifikation mit dem Heimatland respektive dem Einwanderungsland zu haben. Im Gegensatz zu den sonst üblichen Gepflogenheiten in der Wissenschaft findet auch keinerlei Diskussion über Kausalitäten statt, die den Mustern der Verteilung verschiedener Variablen zugrunde liegen.

Es gibt allerdings auch Studien, die zu entgegengesetzten Resultaten hinsichtlich der Bedeutung von Religion und Kultur und des Kampfes der Kulturen für die Integration von Migranten kommen (Manning/Roy 2007; Soroka et al. 2007).

\subsection{DIE NACHFRAGESEITE}

Die empirische Forschung über die Nachfrageseite hat sich verschiedener Methoden bedient, um herauszufinden, ob die Rekrutierungspolitik der Arbeitgeber von deren Vorstellungen und Auffassungen über die Kompetenz verschiedener Gruppen von Individuen beeinflusst wird.

In einer Vielzahl von Studien zu Jugendlichen, die entweder alle in Schweden geboren sind oder vor dem Schulbesuch nach Schweden kamen, gibt es starke Hinweise auf Diskriminierung (Arai et al. 2000; Vilhelmsson 2002; Behtoui 2006b; Nekby et al. 20072). Obwohl diese Studien Jugendliche mit identischen Noten in der schwedischen Sprache vergleichen, zeigen die Resultate, dass Jugendliche außereuropäischer Herkunft seltener Beschäftigung finden und/oder niedrigere Löhne erhalten als Jugendliche mit schwedischem Hintergrund.

In jüngster Zeit sind diese starken Hinweise auf Diskriminierung von neuen Untersuchungen bestätigt worden. Arai/Skogman Thoursie (2007) analysierten die Einkommensentwicklung von Personen, die ihren ausländisch klingenden Nachnamen mit einen schwedisch klingenden getauscht haben. Die Ergebnisse zeigen, dass die Einkommen der Namenstauscher bedeutend schneller wachsen, als die derjenigen, die ihren ursprünglichen Namen beibehalten haben.

Eriksson/Lagerström (2007) verwendeten Daten der „Arbeitsplatzbank“, die von der Arbeitsvermittlung im Internet eingerichtet wurde und in der Arbeitsuchende ihre Profile hinterlegen können. Die Autoren fanden heraus, dass, unabhängig von Ausbildung oder Berufserfahrung, Arbeitsuchende mit nicht-nordischen Namen beinahe ein Drittel weniger Antworten von Arbeitgebern auf ihre Bewerbungen bekommen als Arbeitsuchende mit schwedischen Namen.

Ein anderer Forschungsansatz stützt sich auf die Untersuchung von Verhaltensmustern von Arbeitgebern im Rekrutierungsprozess von Arbeitskräften. Drei vorliegende schwedische Studien vergleichen Jugendliche, von denen die eine Gruppe schwedische Namen und die andere fremde Namen hatte, die eine Herkunft aus dem Vorderen Orient oder Afrika nahelegten. ${ }^{3}$ Die drei Studien zeigen übereinstimmende Resultate. Die Jugendlichen mit einem ausländisch klingenden Namen werden wesentlich seltener zum Bewerbungsgespräch gebeten als ihre Pendants mit gleichen Profilen/Qualifikationen, aber mit schwedischen Namen (Carlsson/Rooth 2006; Bursell 2007; Taran 2007).

\subsection{INSTITUTIONELLE INEFFEKTIVITÄT}

Die Chance für Einwanderer, Arbeit zu finden und vor allem eine ihrer Qualifikation entsprechende, ist nicht nur abhängig von der Angebots- und Nachfrageseite des Arbeitsmarktes. Ebenfalls von Bedeutung sind die institutionellen Rahmenbedingungen und die Einwanderungs- und Integrationspolitik.

In politischen Erklärungen insistiert man schon lange darauf, dass der Arbeitsmarkt im Fokus der Integrationspolitik stehen muss. Der einzelne Einwanderer wird jedoch noch immer allzu oft als Problemfall gesehen: Er soll von Fachleuten im Umgang mit Menschen, die Probleme haben, bearbeitet, motiviert und vorbereitet werden. Eine Perspektive, die Immigranten als handlungsfähige und in vielen Fällen hoch qualifizierte Personen ernst nimmt und damit als Bereicherung für den schwedischen Arbeitsmarkt begreift, fehlt weitgehend.

Unter den wichtigsten integrationspolitischen Maßnamen sind die Einführungsprogramme für neu angekommene Einwanderer. Leider fehlen statistische Daten, die eine systematische Verfolgung und Auswertung der Effekte solcher Programme ermöglichen. Es gibt jedoch Hinweise auf ernste Mängel bei Einführungsprogrammen und Schwedischkursen für Einwanderer. Nur etwa ein Drittel der Teilnehmer an den Sprachkursen weist nach zwei Jahren Unterricht ausreichende Kenntnisse auf. Ein weiteres, seit vielen Jahren bekanntes Problem mit den Einführungsprogrammen besteht darin, dass die schulische Unterrichtung in der schwedischen Sprache allzu selten mit der Arbeitspraxis oder anderen Aktivitäten kombiniert wird, obwohl

\footnotetext{
Diese Studien berücksichtigen Daten über die Ausbildung und/oder den sozioökonomischen Status der Eltern.

3 Der methodische Ansatz: Zwei Personen mit gleichwertigem Profil oder gleichwertiger Qualifikation auf Basis des schwedischen Schulsystems und des schwedischen Arbeitsmarktes bewerben sich um den gleichen Arbeitsplatz; einziger Unterschied ist, dass einer einen schwedisch klingenden, der andere einen ausländisch klingenden Namen führt.
} 
der Lehrplan und die entsprechenden Abkommen zwischen den Kommunen und den staatlichen Behörden dies vorschreiben.

Nach abgeschlossenem Einführungsprogramm finden nur wenige Einwanderer direkt einen Arbeitsplatz. Anstelle von Arbeit folgen in der Regel weitere Kurse und Arbeitsmarktprogramme. Auch hier und insbesondere bei kommunalen Aktivitäten fehlt Evaluation. Einzelne Hinweise deuten jedoch auf mangelhafte Effektivität der Programme.

Die Verfahren zur Anerkennung und Bewertung ausländischer Bildungsabschlüsse und Ausbildungen sind mühsam und intransparent. Dies führt in vielen Fällen da$\mathrm{zu}$, dass auch hochqualifizierte Personen ihre Ausbildungen oder Studien von vorne beginnen müssen. Im Zusammenspiel zwischen Kommunen und Behörden gibt es darüber hinaus gegensätzliche Zielvorstellungen. Dabei geraten die Voraussetzungen und Interessen der arbeitslosen Immigranten in den Hintergrund gegenüber dem Interesse von Kommunen und Behörden an Kostensenkung und Verbesserung ihrer Statistiken. Dadurch entsteht eine Art „Warteraumsyndrom“. Der Einwanderer wird zwischen den Akteuren hin und her geschoben und mit Programmen und Kursen beschäftigt, in der Erwartung, dass er schließlich ausreichend gerüstet sein wird für den Eintritt in den Arbeitsmarkt.

Das Gesamtbild der Integrationspolitik und ihrer Institutionen ist somit wenig überzeugend. Die Defizite betreffen Akteure auf verschiedenen Ebenen, die keinen Anreiz zur Zusammenarbeit verspüren. Es fehlen zentrale Steuerungsmechanismen, die dafür sorgen könnten, dass die übergeordneten integrationspolitischen Zielsetzungen in der Praxis umgesetzt werden.

\section{Was verändert werden sollte}

Ein durchgängiges Phänomen ist das Fehlen fundierter Wirkungsforschung, die man zur Grundlage für Veränderungen der verschiedenen Programme nehmen könnte (Integrationsverket 2006). Ein erster Schritt zur Verbesserung der Effektivität wäre die Verpflichtung derjenigen, die Kurse abhalten, über ihre Tätigkeit eine qualifizierte Statistik zu führen. Besondere Bedeutung kommt hier der Bewertung der Kurse in berufsspezifischem Schwedisch zu. ${ }^{4}$

Ein weiteres Feld sind die Bekämpfung von Diskriminierungen und der Ausgleich für den bei Einwanderern beobachteten Mangel an Netzwerk- und Arbeitgeberkontakten. Eine staatliche Untersuchung zur Diskriminierung (SOU 2005) schlägt vor, in die Verfassung die Verpflichtung der Behörden aufzunehmen, diskriminierende Vorkommnisse in der Ausübung ihrer Tätigkeit zu bekämpfen und eine Anti-Diskriminierungsklausel bei öffentlichen Ausschreibungen einzuführen.

Schweden ist ein Land, in dessen Arbeitsbeziehungen Tarifverträge mindestens so wichtig sind wie die Gesetzgebung. Deshalb hat der Ombudsmann gegen Diskriminierung wiederholt vorgeschlagen, in die Tarifverträge Bestimmungen aufzunehmen, die sich gegen Diskriminierung richten. Vermutlich wäre es ein kraftvolles Instrument, wenn hunderttausende von Vertrauensleuten der Gewerkschaftsbewegung aktiv an ihren Arbeitsplätzen gegen Diskriminierung vorgehen würden und wenn diese Arbeit auch noch tarifvertraglich abgesichert wäre.

Schließlich ist Schweden ein Land mit einer beinahe hundertjährigen Tradition aktiver Arbeitsmarktpolitik. Im Unterschied zu anderen Politikfeldern werden hier die Effekte der arbeitsmarktpolitischen Programme auch evaluiert.

Ein noch laufender Modellversuch für Neuankömmlinge kombiniert eine intensivere Einarbeitung und Beratung mit der Möglichkeit, die Löhne zu subventionieren. Die Auswertung dieses Programms zeigt positive Effekte hinsichtlich der Chancen, später eine Arbeit zu finden (Åslund/Johansson 2006). Das Programm enthält Bestandteile, die einigen Hindernissen auf dem Arbeitsmarkt entgegenwirken. Intensivierte Beratung heißt, dass die Kontakte des Arbeitsvermittlers mit den Arbeitgebern an die Stelle dessen treten können, was vielen im Ausland Geborenen fehlt, nämlich Erfolg versprechende Netzwerke. Die einfache Subventionierung von Löhnen kann zumindest der Art der Diskriminierung entgegenwirken, die sich daraus ergibt, dass Arbeitgeber ihre Anstellungspolitik auf stereotype Auffassungen über gewisse Migrantengruppen und deren vermeintliche Produktivität gründen.

\section{Rolle der Integrations- politik für das schwedische Modell}

Für einen Arbeitsmarktökonomen ist das schwedische Modell das Programm für Vollbeschäftigung, schnellen Strukturwandel, gerechte Löhne und niedrige Inflation, das die Ökonomen Gösta Rehn und Rudolf Meidner in den 1950er Jahren entwickelten (Erixon 2000; Milner/Wadensjö 2001). Die Hauptbestandteile des Rehn-Meidner-Modells waren eine rigorose Finanzpolitik zur Bekämpfung der Inflation und eine solidarische Lohnpolitik zur Vermeidung inflationärer Tendenzen durch zu große Lohnunterschiede. Unter solidarischer Lohnpolitik verstand man gleichen Lohn für gleiche Arbeit. Eine solche Politik führte dazu, dass Unternehmen mit geringer Produktivität aus dem Markt gedrängt wurden, während solche mit hoher Produktivität begünstigt wurden. Diese Bestandteile wurden komplettiert durch eine selektive Arbeitsmarktpolitik, um die Arbeitslosigkeit zu verhindern, die trotzdem noch entstehen kann, aber auch um die Mobilität auf dem Arbeitsmarkt zu erhöhen, sodass die Übertragung von Ressourcen von niedrigproduktiven auf hochproduktive Unternehmen erleichtert wird. Mobilitätsstimulierende Elemente waren dabei Weiterbildungsangebote und Umzugshilfen.

Das Modell betonte ferner das Auseinanderhalten von Sozialpolitik und Arbeitsmarktpolitik, was seit den 1930er Jahren eine schwedische Tradition ist. Nach diesem Verständnis ist Arbeitsmarktpolitik ein Teil der Wirtschaftspolitik. Die Sozialpolitik hingegen soll ein letztes Auffangnetz spannen, für die Menschen, die dauerhaft oder vorübergehend dem Arbeitsmarkt nicht zur Verfügung stehen. Die Grundlagen dieses Modells prägen die schwedische Politik bis heute.

\footnotetext{
4 Als Beispiele seien das fachspezifische Schwedisch der Krankenpflege für eingewandertes Krankenpflegepersonal sowie technisches Schwedisch für eingewanderte Ingenieure genannt. Wenn diese berufsspezifischen Kurse sich als schneller Weg zum Spracherwerb und zum Arbeitsmarkt innerhalb des eigenen Berufes erweisen sollten, scheint es angebracht, diese Erfahrungen auf andere Berufe auszudehnen.
} 
Gösta Rehn beschrieb das Modell einmal als „die Sicherheit der Flügel anstelle der Sicherheit des Schneckenhäuschens". Damit war gemeint, dass die Sicherheit des Individuums in Zeiten strukturellen Wandels in der Chance liegt, Neues zu lernen und neue Arbeitsplätze mit neuen Arbeitsinhalten angeboten zu bekommen. Einwanderer in Schweden kann man dagegen besser als in ihrem schwer beweglichen Schneckenhaus Eingeschlossene beschreiben.

Immigrations- oder Integrationspolitik waren nie Bestandteile oder Handlungsfelder für das oben beschriebene schwedische Modell, und sie werden auch heute noch nicht unter einer solchen Perspektive diskutiert. Man könnte allerdings argumentieren, dass die Politik bezogen auf Arbeitsmigration bis in die 1970er Jahre mit dem Modell vereinbar war, da sie Wachstumsindustrien mit knappen Ressourcen (Arbeitskräften) versorgte. Es könnte weiterhin argumentiert werden, dass die Integrationspolitik, die seit Mitte der 1980er Jahre praktiziert wurde, nicht mit dem Modell vereinbar ist. Immigranten werden seitdem nicht als Ressourcen, sondern als Problemfälle begriffen, um die sich die Sozialpolitik kümmern muss.

Fazit

Zusammenfassend kann festgestellt werden, dass die international gepriesene schwedische Integrationspolitik gemessen an ihrer universalistischen Zielvorstellung, nämlich gleiche Rechte, Möglichkeiten und Pflichten zu gewähren, bisher kein Erfolgsmodell ist. Ihre Mängel liegen dabei sowohl im Bereich der Instrumente der Integrationspolitik (Sprachkurse, Evaluierungen, Netzwerke etc.) als auch in ihrer institutionellen Ausgestaltung und ihrer Einordnung in die Gesamtheit der schwedischen Politik als Sozialpolitik.

Von zentraler Bedeutung für den Misserfolg sind ferner offenkundige Momente der Diskriminierung von Immigranten. Dagegen können Studien, die propagieren, dass Einwanderer aus außereuropäischen Ländern, besonders Muslime, nicht zu westlichen Arbeitsmärkten passen, als empirisch wenig seriös betrachtet werden. Sie bestätigen vielmehr alte Vorurteile. Es gibt keinerlei empirische Belege für die Behauptung, dass Religion und Kultur Hindernisse für die Integration sind.

Die Verlagerung der Zuständigkeit für neu angekommene Flüchtlinge von der Arbeitsmarktpolitik in die Sozialpolitik Mitte der 1980er Jahre bedeutete einen Paradigmenwechsel im Umgang mit den Immigranten. In der Praxis zeigt sich, dass seitdem die Kommunen und in vielen Fällen deren Sozialämter die Integrationspolitik umsetzen. Die problematischen Erfahrungen mit dieser Zuordnung der Integrationspolitik legen nahe, dass es Erfolg versprechender sein dürfte, Integrationspolitik als Teil der Arbeitsmarktpolitik zu betreiben, unabhängig davon, ob die Mi- granten als Arbeitskräfte oder als Flüchtlinge kommen. Das könnte man aus der schwedischen Erfahrung lernen. Zur Veränderung der schwedischen Integrationspolitik in diese Richtung wird es notwendig sein, die verschiedenen Komponenten des Politikfeldes zusammenzuführen, damit alle beteiligten Institutionen zum Erreichen des gewünschten Ziels beitragen können.

Die vielleicht wichtigste Voraussetzung für eine bessere Integration von Migranten in Schweden dürfte aber ein grundsätzlicher Perspektivwechsel sein. Die Immigranten sollten als wertvolle Zukunftsressource, als Bereicherung des Arbeitsmarktes gesehen werden, nicht als soziale Problemfälle. Ein solcher Wechsel der Perspektive könnte bedeuten, dass die Integrationspolitik wieder integraler Bestandteil des schwedischen Arbeitsmarktmodells wird. In ihm verfolgt die aktive Arbeitsmarktpolitik das Ziel, Ressourcen von schwachen auf expandierende Branchen zu übertragen. Für den Arbeitslosen soll dabei das Prinzip der „Sicherheit der Flügel“ gelten, ermöglicht durch Programme, die seine Kompetenz stärken und ihn für expandierende Arbeitsmärkte attraktiv machen. In der Integrationspolitik gilt jedoch im Augenblick in der Hauptsache die "Sicherheit des Schneckenhäuschens", die wohl eine gewisse materielle Sicherheit vermittelt, aber nicht zu Arbeit und Integration in die Gesellschaft führt. 
Arai, M./Schröder, L./Vilhemsson, R. (2000): En svartvit arbetsmarknad en ESO-rapport om vägen från skola till arbete. Expertgruppen för studier i offentlig ekonomi (ESO), Ds 47.

Arai, M./Regnér, H./Schröder, L. (1999): Är arbetsmarknaden öppen för alla? Bilaga 6 till Långtidsutredningen 1999/2000, SOU 7

Arai, M./Skogman Thoursie, P. (2007): Giving up foreign names. An empirical examination of surname change and earnings, Stockholm University Linnaeus Center for Integration Studies (SULCIS), Working Paper 01, Åslund, O./Johansson, P. (2006): Virtues of SIN - effects of an immigrantworkplace introduction program, The Institute for Labour Market Policy Evaluation (IFAU), WP 7

Behtoui, A. (2006a): Unequal Opportunities, akademisk avhandling, Linköping Studies in Arts and Science 369

Behtoui, A. (2006b): Om de hade på rätt plats födda föräldrar. Om ungdomar med utländsk bakgrund i det svenska utbildningssystemet och på den svenska arbetsmarknaden, expertbilaga till Rapport Integration 2005, Norrköping

Bisin, A./Patacchini, E./Verdier, T./Zenou, Y. (2007): Are Muslim Immigrants Different in Terms of Cultural Integration?, Institute for the Study of Labor (IZA), Discussion Paper 3006

British Council/Migration Policy Group (2007): Migration Integration Policy Index, Brussels

Broomé P./Bäcklund A.-K./Lundh, C. /Ohlsson, R. (1996): Varför sitter brassen på bänken?, eller varför har invandrarna så svårt att få jobb? SNS förlag, Stockholm

Bursell, M. (2007): What's in a name? A field experiment test for the existence of ethnic discrimination in the hiring process, Stockholms universitets Linnécentrum för integrationsstudier (SULCIS), Working Paper 07 Carlsson, M./Rooth, D.-O. (2006): Evidence of Ethnic Discrimination in the Swedish Labor Market Using Experimental Data, Institute for the Study of Labour (IZA), Discussion Paper 2281

Constant, A./Gataullina, L./Zimmermann, K. F. (2006b): Gender, Ethnic Identity and Work, Institute for the Study of Labor (IZA), Discussion Paper 2420

Constant, A./Gataullina, L./Zimmermann, K. F./Zimmermann, L. (2006a): Clash of Cultures: Muslims and Christians in the Ethnosizing Process, Institute for the Study of Labor (IZA), Discussion Paper 2350 Edin, P.-A./Fredriksson, P./Åslund, O. (2004): Settlement policies and the economic success of immigrants, in: Journal of Population Economics 17, S. $133-155$

Eriksson, S./Lagerström, J. (2007): Detecting discrimination in the hiring process: evidence from an Internet- based search channel, Institutet för arbetsmarknadspolitiska utvärderingsstudier, Working Paper 19 Erixon, L. (2000): The "Third Way" Revisited. A Revaluation of the Swedish Model in the Light of Modern Economics, Trade Union Institute for Economic Research (FIEF), Working Paper 159

Huntington, S. (2002): The Clash of Civilizations, And the Remaking of World Order, New York
Integrationsverket (2006): Rapport Integration 2005, Norrköping Manning, A./Roy, S. (2007): Culture Clash or Culture Club? The Identity and Attitudes of Immigrants in Britain, Centre for Economic Performance (CEP), Discussion Paper 790

Milner, H./Wadensjö, E. (red) (2001): Gösta Rehn, the Swedish Model and labour market policies, Ashgate

Nekby, L./Vilhelmsson, R./Özcan, G. (2007): Do Domestic Educations Even Out the Playing Field? Ethnic Labor Market Gaps in Sweden, the Stockholm University Linnaeus Center for Integration Studies (SULCIS), Working Paper 3

OECD (2004): The integration of immigrants into the labour market. The case of Sweden. Swedish Review, DELSA/ELSA/(2004)13, Paris

OECD (2005): The labour market integration of immigrants in Germany, DELSA/ELSA/WP2 (2005)3, Paris

OECD (2007): International Migration Outlook, SOPEMI 2007, Paris Olli-Segendorf, Å. (2005): Job Search Strategies and Wage Effects for Immigrants, Institutet för social forsknings avhandlingsserie 65, Stockholm

Rooth, D.-O./Åslund, O. (2006): Utbildning och kunskaper i svenska. Framgångsfaktorer för invandade? SNS förlag, Stockholm Rosholm, M./Scott, K./Husted, L. (2001): The Times They Are A-changing. Organizational Change and Immigrant Opportunities in Scandinavia, The Institute for the Study of Labour (IZA), Discussion Paper 258

Schröder, L. (2007): From problematic objects to resourceful subjects: An overview of immigrant-native labour market gaps from a policy perspective, in: Swedish Economic Policy Review 14, S. 7-31

Scott, K. (1999): The Immigrant Experience: Changing Employment and Income Patterns in Sweden 1970-1993, Lund Studies in Economic History 9

Soroka, S. N./Johnston, R./Banting, K. (2007): Ties That Bind? Social Cohesion and Diversity in Canada, in: Banting, K./Courchene, T. J./ Seidle, L. (Hrsg.): The Art of the State III: Belonging? Diversity, Recognition and Shared Citizenship in Canada, Institute for Research in Public Policy, Montreal

Statens offentliga utredningar (SOU) (2005): Det blågula glashuset, SOU 56 , Stockholm

Statistiska centralbyrån (2008) Statistisk årsbok 2008, Stockholm Taran, P. (2007): Discrimination against native Swedes of immigrant origin in access to employment, in: International Migrations Papers 2, International Labour Office, Geneva

Vilhemsson, R. (2002): Wages and Unemployment of Immigrants and Natives in Sweden, Institutet för social forsknings avhandlingsserie 56 Widgren, J. (1987): Svensk invandrarpolitik, Liber, Malmö

Zimmermann, L./Gataullina, L./Constant, A./Zimmermann, K. F. (2006): Human Capital and Ethnic Self-Identification of Migrants, Institute for the Study of Labor (IZA), Discussion Paper 2300 